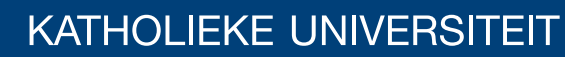 \\ LEUVEN
}

\section{Faculty of Business and Economics}

\section{Profit-based latency problems on the line}

Sofie Coene, Frits C.R. Spieksma

DEPARTMENT OF DECISION SCIENCES AND INFORMATION MANAGEMENT (KBI) 


\title{
Profit-based Latency Problems on the Line
}

\author{
Sofie Coene* Frits C.R. Spieksma*
}

\begin{abstract}
The latency problem with profits is a generalization of the minimum latency problem. In this generalization it is not necessary to visit all clients, however, visiting a client may bring a certain revenue. More precisely, in the latency problem with profits, a server and a set of $n$ clients, each with corresponding profit $p_{i}(1 \leq i \leq n)$, are given. The single server is positioned at the origin at time $t=0$ and travels with unit speed. When visiting a client, the server receives a revenue of $p_{i}-t$, with $t$ the time at which the server reaches client $i(1 \leq i \leq n)$. The goal is to select clients and find a route for the server such that total collected revenue is maximized. We formulate a dynamic programming algorithm to solve this problem when all clients are located on a line. We also consider the problem on the line with $k$ servers and prove NP-completeness for the latency problem on the line with $k$ non-identical servers and release dates. In this proof we also settle the complexity of an open problem in de Paepe et al. [4].
\end{abstract}

Key words: minimum latency; traveling repairman; dynamic programming; complexity

\section{Introduction}

Consider the following problem. Given are a set of $n$ clients located in some metric space and profits $p_{i}$ associated with each client $i, 1 \leq i \leq n$. In addition, a single server is given, positioned at the origin at time $t=0$. The server travels at unit speed. If the server serves client $i$ at time $t$, the revenue collected by the server equals $p_{i}-t$. The goal is to select clients and to find a route for the server serving the selected clients, such that total collected revenue is maximal. We refer to this problem as the traveling repairman problem with profits, or TRPP for short. In this paper we restrict ourselves to the line as a metric space. Notice that in the TRPP (i) not every client needs to be served, and (ii) the revenue collected at a client depends on the time needed to reach that client.

In this paper we show:

- how a dynamic program solves the TRPP on the line in polynomial time, thereby generalizing a classical result from Afrati et al. [1],

- how this result can be generalized to the problem with multiple identical servers (referred to as MTRPP on the line),

*Katholieke Universiteit Leuven, Operations Research Group, Naamsestraat 69, B-3000 Leuven, Belgium. 
- that the problem with multiple non-identical servers and release dates for each client, is NP-hard.

In the proof of the latter result we settle the complexity of an open problem mentioned in de Paepe et al. [4].

The paper is organized as follows. In Section 2 we describe the literature for the traveling repairman problem and we motivate the TRPP on the line. In Section 3 we describe the dynamic programming algorithm. We settle the complexity of different variants of MTRPP in Section 4. In the 5 th and last section we state some open problems.

\section{Literature and Motivation}

The TRPP is a generalization of the well-known traveling repairman problem (TRP), also known as the minimum latency problem (MLT). In this problem, no profits are given and the goal is to serve all clients with minimal total latency. Afrati et al. [1] give an $O\left(n^{2}\right)$ dynamic program for the TRP on the line which was later improved to $O(n)$ by García et al. [7]. In [12] it is proven that the TRP on the line with release dates is (weakly) NPhard. Minieka [9] shows that the problem on a tree network is polynomial for trees with unit weights and develops a polynomial time algorithm for the problem on weighted trees when the number of leaves is bounded. The TRP on weighted trees is proven to be strongly NP-hard by Sitters [11]. The problem on the line with multiple identical servers is solvable in $O\left(n^{4}\right)$, see $\mathrm{Wu}[13]$ and Averbakh and Berman [2]. We refer to $\mathrm{Wu}$ [14] et al. and the references contained therein for papers dealing with exact algorithms for the TRP (i.e. the problem with an arbitrary metric space).

In de Paepe et al. [4] a framework is described dealing with the computational complexity of dial-a-ride problems (which include latency problems, and in particular latency problems on the line). However, as far as we are aware there is no work on latency problems with profits. This is in contrast with the situation for the traveling salesman problem (TSP), see Feillet et al. [5] for a survey on the TSP with profits.

\section{Motivation}

As indicated above, a variety of latency problems arise in many different settings. However, a common characteristic is the focus on minimizing total waiting time of the clients. Here we consider a profit-oriented objective. In situations where a server receives some revenue by performing a service, this objective can be more appropriate. Of course, one needs to balance in some way the waiting times and the profits. This can be done in various ways (bounding the total waiting time by a constant from above, bounding the total revenue realized by a constant factor from below). Here, however, we propose to combine profit and latency in a single objective (as described in the introduction). By doing so, it is clear that a latency problem with a profit objective is at least as hard as the corresponding "ordinary" latency problem.

We restrict the analysis in this paper to a linear profit function (i.e. $p_{i}-t$ ) in the objective function. We use this particular function because it arises in the pricing problem of an integer programming formulation modeling the latency problem with multiple servers. Indeed, 
consider a situation with $K$ nonidentical servers (meaning that their speed may differ) whose job is to service a set of $n$ clients on the line. We now describe a set-partitioning formulation for this problem. We define $c_{r k}$ as the total latency of a feasible route $r(r=1, \ldots, R$; with $R$ the number of feasible routes) served by server $k(k=1, \ldots, K)$. Then, variable $x_{r k}$ is equal to 1 if route $r$ is served by server $k$ and 0 otherwise.

$$
\begin{aligned}
\text { Minimize } & \sum_{r=1}^{R} \sum_{k=1}^{K} C_{r k} x_{r k} \\
\text { subject to } & \sum_{r: i \in r} \sum_{k=1}^{K} x_{r k}=1 \quad \text { for } i=1, \ldots, n ; \\
& \sum_{r=1}^{R} x_{r k} \leq 1 \quad \text { for } k=1, \ldots, K ; \\
& x_{r k} \in\{0,1\} .
\end{aligned}
$$

The objective is to minimize total latency (1), there is a constraint for each client stating that it must be served exactly once (2) (see also [6]), and every server can be used at most once (3). When solving the linear programming relaxation of this integer program, the dual variables $u_{i}(i=1, \ldots, n)$ corresponding to constraints $(2)$ act as the profits in the resulting pricing problem. Indeed, verifying for a fixed server $k$ whether a violated dual constraint exists, amounts to minimizing $C_{r k}-\sum_{i: i \in r} u_{i}$. This corresponds to the objective function in the TRPP.

In this paper we consider the line-metric. Practical examples where this metric is relevant are e.g. "shoreline"-problems [10]. These problems arise when scheduling and routing cargoships to visit a number of ports which are usually located along a shoreline. Friese and Rambau [6] describe a setting with multiple elevators who need to serve a set of requests.

Summarizing, the TRPP is interesting because (i) it is a first attempt to combine a latency objective with profits, (ii) latency problems on the line are relevant and their complexity is often unresolved [4], (iii) the TRPP occurs as the pricing problem of an integer programming formulation modeling the latency problem with multiple servers, as is explained above.

\section{A Polynomial Algorithm for the TRPP}

We represent an instance of the TRPP on the line as depicted in Figure 1. The server starts in the origin $x_{0}=y_{0}=0$. In this section, we refer to the clients left of the origin as $x_{1}, x_{2}, \ldots, x_{r}$, and to the clients right of the origin as $y_{1}, y_{2}, \ldots, y_{q}$. To each client a profit $p_{x_{i}}$ resp. $p_{y_{j}}$ (with $\left.i=0, \ldots, r ; j=0, \ldots, q\right)$ is associated. Notice that we sometimes identify a client with its position on the line. We make a distinction between "served" clients and "visited" clients. A client is served when a server has performed a service at that client and has collected profit there; when the server passes a client without performing the service, this client has been visited but not served. The goal is to select clients and to find a route for the server such that total profits of the clients served minus the latency of the corresponding route is maximal. Clearly, the TRPP on the line generalizes the traveling repairman problem on the line: in case each of the profits is huge, it is optimal to serve all clients, 


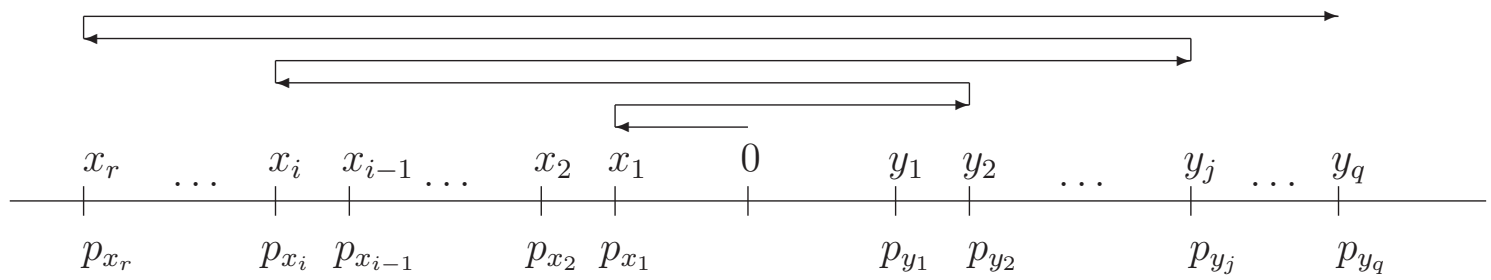

Figure 1: The TRPP on the line

and the problem reduces to finding a route with minimal latency. In general however, not every client needs to be served in the TRPP; observe however that, for those clients that are served, it is optimal to visit the client the first time the server passes by. The optimal route thus has a spiral shape as illustrated in Figure 1 (see [4]).

Given an instance of TRPP it is not yet clear which clients, and in particular how many clients, need to be selected. We deal with this issue by proposing a procedure that keeps track of the number of clients to be visited.

We now define the ingredients of our dynamic program (DP). A state in our DP is denoted by $\left[x_{i}, y_{j}, l\right]$ which corresponds to the situation where the server is positioned in $x_{i}$ (as its leftmost visited client), where the rightmost visited client is $y_{j}$, and where $l$ clients are to be served outside that interval $(0 \leq i \leq r, 0 \leq j \leq q, 1 \leq l \leq r+q)$. Similarly, in the state $\left[y_{j}, x_{i}, l\right]$ the server is at position $y_{j}$ (as its rightmost visited client), the leftmost visited client is $x_{i}$, and $l$ clients will be served outside this interval.

\section{Definition 1}

$\forall i, j, l: P\left[x_{i}, y_{j}, l\right]$ equals the maximal value of the difference between

(i) the profits of the clients served in $\left[x_{i}, y_{j}\right]$, and

(ii) the latency costs incurred for these clients served in $\left[x_{i}, y_{j}\right]$ taking into account that $l$ clients will be served outside this interval.

We refer to $P\left[x_{i}, y_{j}, l\right]$ as the revenue.

Notice that the route followed by the server after having reached state $\left[x_{i}, y_{j}, l\right]$ does not depend on the route followed within $\left[x_{i}, y_{j}, l\right]$. Thus, only the set of clients yielding a maximal value for $P\left[x_{i}, y_{j}, l\right]$ will be selected in an optimal solution. This observation is instrumental for the correctness of our DP algorithm, that we will now describe. First, we define $t\left[x_{i}, y_{j}\right]$ as the distance between clients $x_{i}$ and $y_{j}$ (for all $i, j$ ). In an initialization step we set the revenue in all states equal to $-\infty$; then we compute the revenue in a state as follows.

For $l=1, \ldots, r+q$ : 


$$
P\left[x_{0}, y_{0}, l\right]=P\left[y_{0}, x_{0}, l\right]=0 .
$$

For $i=0, \ldots, r ; j=0, \ldots, q ; l=1, \ldots, r+q$ :

$$
\begin{aligned}
& P\left[x_{i}, y_{j}, l\right]=\max \{ \\
& P\left[x_{i-1}, y_{j}, l+1\right]+p_{x_{i}}-(l+1) * t\left[x_{i-1}, x_{i}\right], \\
& P\left[x_{i-1}, y_{j}, l\right]-(l) * t\left[x_{i-1}, x_{i}\right], \\
& P\left[y_{j}, x_{i-1}, l+1\right]+p_{x_{i}}-(l+1) * t\left[y_{j}, x_{i}\right], \\
& \left.P\left[y_{j}, x_{i-1}, l\right]-(l) * t\left[y_{j}, x_{i}\right] \quad\right\} \\
& P\left[y_{j}, x_{i}, l\right]=\max \{ \\
& P\left[y_{j-1}, x_{i}, l+1\right]+p_{y_{j}}-(l+1) * t\left[y_{j-1}, y_{j}\right], \\
& P\left[y_{j-1}, x_{i}, l\right]-(l) * t\left[y_{j-1}, y_{j}\right], \\
& P\left[x_{i}, y_{j-1}, l+1\right]+p_{y_{j}}-(l+1) * t\left[x_{i}, y_{j}\right], \\
& \left.P\left[x_{i}, y_{j-1}, l\right]-(l) * t\left[x_{i}, y_{j}\right] \quad\right\} .
\end{aligned}
$$

Observe that $P\left[x_{0}, y_{j}, l\right]=P\left[y_{0}, x_{i}, l\right]=-\infty$ for $i>0$ and $j>0$. Then total revenue is:

$$
\text { TotalRevenue }=\max \left\{\max _{i, j}\left\{P\left[x_{i}, y_{j}, 0\right]\right\}, 0\right\} .
$$

Theorem 1 Algorithm DP is correct.

Proof. We establish correctness of (4) (the arguments for (5) are similar) by using induction on $i$ for a fixed $j$, thereby proving Theorem 1 . Correctness of (4) is shown by arguing that it leads to values for $P\left[x_{i}, y_{j}, l\right]$ that satisfy Definition 1 . In case $i=0$, we already observed that $P\left[x_{0}, y_{j}, l\right]=-\infty$ (which is in agreement with Definition 1 ).

We will use induction and assume that $P\left[x_{i-1}, y_{j}, l+1\right]$ and $P\left[x_{i-1}, y_{j}, l\right]$ satisfy Definition 1 . The question now is whether $P\left[x_{i}, y_{j}, l\right]$ computed using (4) satisfies Definition 1. Consider the revenue realized when the server is positioned in $x_{i}$, while $y_{j}$ is the rightmost visited client. We distinguish two cases: $x_{i}$ is served and $x_{i}$ is not served. Consider first the case where $x_{i}$ is served; as it is the last client visited, it is the last client served. Then, revenue can be broken down into three terms: (i) the revenue realized after serving a set of clients in $\left[x_{i-1}, y_{j}\right]$ when $l+1$ clients will be served outside this interval, (ii) the travel time between the previous client visited and $x_{i}$, taking into account the $(l+1)$ clients left to be served, (iii) the profit $p_{x_{i}}$. The previous client visited cannot lay outside $\left[x_{i}, y_{j}\right]$, in fact it is either $x_{i-1}$ or $y_{j}$. Indeed, all clients within $\left[x_{i-1}, y_{j}\right]$ are met before $x_{i}$ and it is optimal to visit them the first time the repairman passes by. Terms (ii) and (iii) are independent of the set of clients served and thus of the revenue realized in state $\left[x_{i-1}, y_{j}, l+1\right]$. As a consequence, only the set of clients yielding the maximal revenue in $\left[x_{i-1}, y_{j}, l+1\right]$ can lead to an optimal 


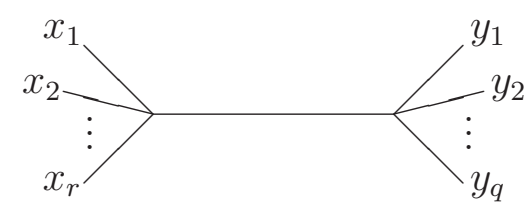

Figure 2: A tree with width three

solution. It follows that the induction hypothesis tells us that the first term is accurately described by $P\left[x_{i-1}, y_{j}, l+1\right]$ or by $P\left[y_{j}, x_{i-1}, l+1\right]$. In addition, the second term equals $(l+1) * t\left[z, x_{i}\right]$ where $z$ denotes the previous client visited $\left(x_{i-1}\right.$ or $\left.y_{j}\right)$, and finally the third term equals $p_{x_{i}}$. Now consider the case where $x_{i}$ is not served. This means that only $l$ clients are left to be served outside $\left[x_{i-1}, y_{j}\right]$ and no profit is realized in $x_{i}$. Revenue can be broken down into two terms: (i) the revenue realized after having served a set of clients in $\left[x_{i-1}, y_{j}\right]$ and $l$ clients will be served outside this interval and (ii) the travel time between the previous client visited $\left(x_{i-1}\right.$ or $\left.y_{j}\right)$ and $x_{i}$, taking into account the $l$ clients left to be served. Again, term (ii) is independent of the set of clients served and thus the revenue realized in state $\left[x_{i-1}, y_{j}, l\right]$. As a consequence, only the set of clients yielding the maximal revenue in $\left[x_{i-1}, y_{j}, l\right]$ can lead to an optimal solution. It follows that the induction hypothesis tells us that the first term is accurately described by $P\left[x_{i-1}, y_{j}, l\right]$ or by $P\left[y_{j}, x_{i-1}, l\right]$. In addition, the second term equals $(l) * t\left[z, x_{i}\right]$ where $z$ denotes the previous client visited.

By taking the maximum of the four terms considered above, we see that $P\left[x_{i}, y_{j}, l\right]$ equals its definition. A similar argument holds for $P\left[y_{j}, x_{i}, l\right]$.

To estimate the complexity of DP with $n=r+q$ : we have at most $n^{3}$ possible states, and since every state has 4 elements in its maximization function, the time complexity of the algorithm is $O\left(n^{3}\right)$, and we can state our result.

\section{Corollary 1 DP solves TRPP on the line in polynomial time.}

Let us consider a number of directions in which Corollary 1 can be extended. First, notice that the distances $t\left[x_{i}, y_{j}\right]$ need not be symmetric. Thus, situations where the line models a river with clients located at upstream and downstream positions are solvable by DP. Next, we can extend Corollary 1 to other geometries. Consider $n$ clients and one server positioned on a circle. Observe that in an optimal solution there is a circle segment between two clients not traversed by the server. Hence, by considering $O(n)$ TRPP instances on the line we can solve the problem on the circle.

We can also extend our result to the TRPP when the clients are positioned on the endpoints of a tree with width three and with positive real lengths on the edges. The observation here is that, in any optimal solution, the spokes at either end of the tree are visited in increasing order of their lengths [3]. Figure 2 shows an example of such a tree with $r$ clients on the left side and $q$ clients on the right side of the tree.

A state in DP $\left[x_{i}, y_{j}, l\right]$ then represents the situation in which $x_{i}$ is the longest spoke visited at the left side and the current position of the server, $y_{j}$ is the longest spoke visited at the right side, $l$ is the number of clients left to be served. Similarly, we can define state $\left[y_{j}, x_{i}, l\right]$ with the current position of the server being $y_{j}$ and $x_{i}$ the longest spoke visited on the other 
side. DP can now be run using the states as defined here.

Thirdly, Corollary 1 can be extended to the TRPP on the line with common release dates. In this variant of the TRPP, a release date $M$ is associated with every client, implying that a client cannot be served before $t=M$. Notice that, if $M$ is large, this is equivalent to choosing a starting location for the server, since then the server can travel and choose the position where it will start at time $t=M$. Of course, in any optimal solution the server, starting at the origin at $t=0$, will at time $M$ either be in $M$ or in $-M$ or at one of the clients in between these two points and wait there until the release date is reached. Thus, the server has at most $n$ choices for its starting position at time $t=M$ and again DP is able to solve this problem.

A fourth extension is the TRPP on the line with constant repair times. Suppose that there is a repair time $h$ at every client which is the same for all clients. This changes the revenue of a route only by a constant factor $\frac{1}{2} S(S+1) h$ where $S$ is the total number of clients visited in the route. Thus, adding constant repair times has no influence on the computational complexity of the TRPP.

Finally, it is interesting to consider different profit functions in the objective, such as e.g. $p_{i}-t^{2}$. In DP, though, the waiting time $t$ of a client $i$ is built up gradually in every iteration. In fact, one can argue that correctness of DP implies $f(x+y)=f(x)+f(y)$, which suggests that DP can only be applied in case of a continuous linear function $f$. A function for which this property holds is $p_{i}-w_{i} t$, with $w_{i}$ the weight of client $i$. DP deals with this weighted version of TRPP by making $w_{i}$ copies of each client $i$, each with a profit equal to $\frac{p_{i}}{w_{i}}$ and with a distance 0 from each other. We solve the resulting instance using DP. An optimal solution will have the property that either all or none of the copies of a client $i$ will be served. Notice that - assuming a binary encoding - this is no longer a polynomial time procedure, but pseudo-polynomial.

\section{The Complexity of MTRPP}

In this section we discuss the TRPP with multiple servers (denoted by MTRPP). Given are the positions of $n$ clients on the line and their associated profits $p_{i}(1 \leq i \leq n)$ and $k$ servers, characterized by a speed $s_{j}(1 \leq j \leq k)$ and a starting location. The goal is to select clients and to find $k$ routes serving each selected client, such that total collected revenue is maximized. Recall that the revenue collected at a client depends on the time needed to reach that client. Observe that, if all servers are in the origin at $t=0$ and if all servers have equal speed, the problem becomes trivial: one server travels to the left and a second server travels to the right, and when they meet a client that contributes positively to the revenue, the client is visited. In case the starting locations of the $k$ servers are arbitrary (but given), and the servers have identical speed, we claim the following:

Theorem 2 MTRPP on the line with multiple identical servers is solvable in polynomial time.

Proof. We only give a short sketch of the proof since it is similar to the proof of $\mathrm{Wu}[13]$ showing polynomial time solvability of the $k$-traveling repairmen problem on the line. First 
of all, note that the servers will never pass by one another because they all have the same speed. A solution to the MTRPP on the line with identical servers thus consists in a division of the line into $k$ consecutive intervals and solving the TRPP on the line for each interval. Consider a line with $n$ clients positioned at $x_{1}, x_{2}, \ldots, x_{n}$ and $k$ servers positioned at $z_{1}, z_{2}, \ldots, z_{k}$. An interval on this line is denoted by $I(i, j)$, containing clients $(i, i+1, \ldots, j)$ with $i \leq j$. Then, define $P(i, j)$ as the maximal revenue of a set of routes visiting clients $(i, i+1, \ldots, j)$ by the repairmen whose origins are within the interval $I(i, j)$. In a first phase compute $P(i, j)$ for each interval $I(i, j)$ containing exactly one origin. This can be done by computing revenue for each server $k$ for its largest possible interval (i.e. the largest interval containing only server $k$ ) using DP. Then, maximal revenue for every smaller interval containing only server $k$ is also known. Indeed, DP computes revenue for all combinations of $i$ and $j$ with $l$ equal to zero. Total time complexity of this first phase is thus $O\left(n^{4}\right)$. In a second phase we select one interval for every server. For $1<i<k$ and $z_{i} \leq m<z_{i+1}$ we compute $P(1, m)=\max _{z_{i-1} \leq j<z_{i}}\{P(1, j)+P(j+1, m)\}$, and total revenue $P(1, n)=$ $\max _{z_{k-1} \leq j<z_{k}}\{P(1, j)+P(j+1, n)\}$. Time complexity of the second phase is $O\left(n^{2}\right)$; total time complexity is thus dominated by the first phase and equal to $O\left(n^{4}\right)$.

However, when the $k$ servers have arbitrary speeds and clients have release dates $r_{i} \geq 0$, we claim that:

Theorem 3 MTRPP on the line with non-identical servers and release dates is strongly NP-hard.

We will prove NP-hardness for the MTRPP with non-identical servers and release dates by settling the complexity of an open problem mentioned in de Paepe et al. [4], i.e. $Q \mid s=$ $t, r_{j} \mid$ line $\mid \sum C_{j}$. This problem is a latency problem $\left(\sum C_{j}\right)$ on the line, with $k$ nonidentical servers $(Q)$ and release dates $\left(r_{j}\right)$; the phrase ' $s=t$ ' refers to the fact that the clients only need to be visited (there is no transportation of clients). Notice that the clients do not have profits and each client needs to be served. The goal is to find routes for every server such that total latency is minimal. Recall that the profit variant of this problem, MTRPP with non-identical servers and release dates, is a generalization of this problem and thus at least as hard.

We transform numerical matching with target sums (NMTS) to $Q\left|s=t, r_{j}\right|$ line $\mid \sum C_{j}$. In an instance of NMTS we are given positive integers $x_{i}(1 \leq i \leq m), y_{j}(1 \leq j \leq m)$ and $b_{l}(1 \leq l \leq m)$. The question is whether there exists a collection of $m$ triples $(i, j, l)$ such that (i) $x_{i}+y_{j}=b_{l}$ for each triple, and (ii) each integer in the input occurs exactly once. This problem is proven to be NP-hard by Garey and Johnson [8].

Proof. We construct an instance of $Q\left|s=t, r_{j}\right|$ line $\mid \sum C_{j}$ by specifying the speeds and the starting location of the servers, and the release dates and the location of the clients as follows. There are $k:=m$ servers, the starting location of each server is the origin, and each server $j$ has speed $b_{j}$ (i.e. $s_{j}=b_{j}, j=1, \ldots, m$ ). There are $2 m$ clients, $m$ clients are located to the left of the origin at $-x_{i}, 1 \leq i \leq m$ (the "left" clients), and $m$ clients are located to the right of the origin at $y_{j}, 1 \leq \bar{j} \leq m$ (the "right" clients). The release date of each left client equals $M \equiv \max _{i} x_{i}$ (i.e., $\left.r_{i}=M\right)$. The release date of each right client equals $M+1$ 


$\begin{array}{ccccccccc}M & & M & M & & M+1 & M+1 & & M+1 \\ -x_{m} & \cdots & -x_{2} & -x_{1} & 0 & y_{1} & y_{2} & \cdots & y_{m}\end{array}$

Figure 3: MTRPP with release dates

(i.e., $r_{j}=M+1$ ). The question is: does there exist a solution to $Q\left|s=t, r_{j}\right|$ line $\mid \sum C_{j}$ such that total latency is equal to $m M+m(M+1)$ ? This completes the description of an instance of $Q\left|s=t, r_{j}\right|$ line $\mid \sum C_{j}$.

We now establish correspondence between the two questions. Clearly, if there exists a numerical matching with target sums, we can direct each server $j$ to the appropriate left location, where it waits till the release date $M$, serves the client at that location, travels to the appropriate right location (as given by the matching), arrives at time $M+1$ (due to the existence of a matching) and serves the right client. Total latency equals $m M+m(M+1)$.

If there exists a solution with total latency $m M+m(M+1)$, it follows that each client must be served at its release date. This implies that the $m$ servers have to be present at $t=M$ at the locations of the $m$ left clients, and at $t=M+1$ the servers need to be present at the $m$ right clients. Hence, each server $j$ travels from a unique left client at $-x_{i}$ to a unique right client at $y_{j}$ in one time unit. It follows that NMTS has a solution.

Corollary $2 Q\left|s=t, r_{j}\right|$ line $\mid \sum C_{j}$ is strongly NP-hard.

Notice that $\mathrm{Yu}$ et al. [15] show that NMTS remains hard even when $x_{i}=i(i=1, \ldots, m)$ and $y_{j}=j(j=1, \ldots, m)$. It follows that the TRPP problem remains hard when the clients are one distance-unit apart.

\section{Open Problems}

The complexity of the following latency problems with profits is open at this moment:

(i) MTRPP with non-identical servers

(Notice also that the complexity of the problem without profits is still open, see [4])

(ii) the weighted TRPP (we showed in Section 4 how DP can be used to solve this problem in pseudo-polynomial time; this however does not settle complexity of weighted TRPP)

(iii) it is unclear whether the $O(n)$ algorithm for the TRP in García et al. [7] could be used for the TRPP. Potentially, this could give a speedup of the current $O\left(n^{3}\right)$ bound of DP.

Acknowledgements: We are grateful to a referee whose remarks led to improved complexity bounds for our DP. 


\section{References}

[1] F. Afrati, S. Cosmadakis, C. H. Papadimitriou, G. Papageorgiou, N. Papakostantinou, The complexity of the traveling repairman problem, Informatique Théorique et Applications 20 (1986) 79-87.

[2] I. Averbakh, O. Berman, Routing and location-routing p-deliverymen problems on a path, Transportation Science 28 (1994) 162-166.

[3] A. Blum, P. Chalasani, D. Coppersmith, B. Pulleyblank, P. Raghavan, M. Sudan, The minimum latency problem, Proceedings of the twenty-sixth annual ACM symposium on the theory of computing (STOC) (1994) 163-171.

[4] W. E. de Paepe, J. K. Lenstra, J. Sgall, R. A. Sitters, L. Stougie, Computer-aided compexity classification of dial-a-ride problems, INFORMS Journal on Computing 16 (2004) 120-132.

[5] D. Feillet, P. Dejax, M. Gendreau, Traveling salesman problems with profits, Transportation Science 39 (2005) 188-205.

[6] P. Friese, J. Rambau, Online-optimization of multi-elevator transport systems with reoptimization algorithms based on set-partitioning models, Discrete Applied Mathematics 154 (2006) 1908-1931.

[7] A. García, P. Jodrá, J. Tejel, A note on the traveling repairman problem, Networks 40 (2002) 27-31.

[8] M. R. Garey, D. S. Johnson, Computers and Intractability: A Guide to the Theory of NP-completeness, Freeman, San Francisco, 1979.

[9] E. Minieka, The delivery man problem on a tree network, Annals of Operations Research 18 (1989) 261-266.

[10] H. N. Psaraftis, M. M. Solomon, T. L. Magnanti, T. Kim, Routing and scheduling on a shoreline with release times, Management Science 36 (1990) 212-223.

[11] R. Sitters, The minimum latency problem is NP-hard for weighted trees, Proceedings of the ninth International Conference on Integer Programming and Combinatorial Optimization (IPCO) LNCS 2337 (2002) 230-239.

[12] R. Sitters, Complexity and Approximation in Routing and Scheduling, PhD thesis, Technical University Eindhoven, 2004.

[13] B. Y. Wu, Polynomial time algorithms for some minimum latency problems, Information Processing Letters 75 (2000) 225-229.

[14] B. Y. Wu, Z. Huang, F. Zhan, Exact algorithms for the minimum latency problem, Information Processing Letters 92 (2004) 303-309. 
[15] W. Yu, H. Hoogeveen, J. K. Lenstra, Minimizing makespan in a two-machine flow shop with delays and unit-time operations is NP-hard, Journal of Scheduling 7 (2004) 333348. 